\title{
UNIVERSITY OF MIAMI RADIOCARBON DATES VI
}

\author{
J J STIPP, K L ELDRIDGE, and R CADWELL
}

Department of Geology, University of Miami, Coral Gables, Florida

The following radiocarbon measurements are a partial list of projects and samples dated since the Spring of 1975. The technique used is liquid scintillation counting of wholly synthesized benzene as indicated in $\mathrm{R}$, $\mathrm{v} 16, \mathrm{p}$ 402-408. The intermediate chemical step of converting $\mathrm{CO}_{2}$ to $\mathrm{Li}_{2} \mathrm{C}_{2}$ has been modified so that the $\mathrm{CO}_{2}$ is reacted with the lithium metal at a temperature of $950^{\circ}$ to $1000^{\circ} \mathrm{C}$ instead of the $600^{\circ} \mathrm{C}$ as formerly done (Tamers, 1975). This modification has had the effect of reducing occasional variable losses in conversion yields in this step, and reducing the reaction time required from $30 \mathrm{~min}$ to $10 \mathrm{~min}$ for a typical $1 / 4$ mole sample. Dates are calculated using a ${ }^{14} \mathrm{C}$ half-life of $5568 \mathrm{yr}$ and errors are reported as one standard deviation. No other correction factors are applied.

ACKNOWLEDGMENTS

We are grateful to D Evans, Dept Biology, for the supplemental use of his Packard Tri-Carb 2003 liquid scintillation spectrometer. We also wish to thank students $\mathrm{P}$ Crevello and $\mathrm{J}$ Hattner for their assistance with various aspects of laboratory preparations.

\section{SAMPLE DESCRIPTIONS}

\author{
I. ARCHAEOLOGIC SAMPLES
}

\section{A. United States}

\section{Arch Creek Shell Midden series}

Shell and charcoal samples from Arch Creek site, Dade Co, Florida $\left(25^{\circ} 08^{\prime} 17^{\prime \prime} \mathrm{N}, 80^{\circ} 10^{\prime} 55^{\prime \prime} \mathrm{W}\right)$. Dates period of midden use by early Florida Indians (R, v 16, p 403-404). Coll July 1975 by R Carr, Florida Div Archives, Tallahassee, Florida; subm July 1975 by W Coleman, Miami West India Archeol Soc, Miami, Florida.

\section{UM-617. Arch Creek 2}

$1730 \pm 80$

Shell (Strombus gigas) from S wall of Pit E55/S13 at $47 \mathrm{~cm}$ depth.

UM-618. Arch Creek 3

$1360 \pm 80$ AD 590

Shell (Strombus gigas) from E wall of Pit E55/S13 at $18 \mathrm{~cm}$ depth.

UM-619. Arch Creek 7

$2320 \pm 80$

Charcoal from floor of Pit E65/S12 at 25 to $35 \mathrm{~cm}$ depth.

UM-620. Arch Creek 9

$2250 \pm 90$

Charcoal from floor of Pit E70/S12 at $25 \mathrm{~cm}$ depth.

$\mathbf{3 0 0} \mathbf{B C}$ 


\section{Wightman series}

Shell and charcoal samples from shell mound of Wightman Site I, Sanibel I., Florida $\left(26^{\circ} 28^{\prime} 18^{\prime \prime} \mathrm{N}, 89^{\circ} 09^{\prime} 16^{\prime \prime} \mathrm{W}\right)$. Coll and subm 1975 by $\mathrm{R} \mathrm{H}$ and $\mathrm{H} \mathrm{H}$ Cadwell.

General Comment (RHC): results indicate a shell mound constructed at different times over a midden. Variation in periods of occupation possibly resulted from sea level fluctuations.

\section{UM-478. Wightman A-1}

$$
1240 \pm 90
$$

Busycon contrarium from 0 to $46 \mathrm{~cm}$ below surface of mound within Grid A.

UM-480. Wightman A-2

$$
1700 \pm 60
$$

Busycon contrarium from 46 to $92 \mathrm{~cm}$ below surface of mound within Grid A.

UM-481. Wightman A-3

$$
1580 \pm 70
$$

Busycon contrarium from 92 to $138 \mathrm{~cm}$ below surface of mound within Grid A.

UM-477. Wightman A-3 and A-4 combined

$$
2250 \pm 80
$$

$\mathbf{3 0 0} \mathbf{~ B C}$

Charcoal from 92 to $184 \mathrm{~cm}$ below surface of mound within Grid A.

UM-482. Wightman A-4

$2050 \pm 160$

Busycon contrarium from 138 to $184 \mathrm{~cm}$ below surface of mound within Grid A.

UM-483. Wightman A-5

$2330 \pm 100$

$380 \mathrm{BC}$

Aequipecten irradians from 184 to $230 \mathrm{~cm}$ below surface of mound within Grid A.

\section{UM-484. Wightman A-6}

$$
2830 \pm 80
$$

880 BC Grid A.

Area ponderosa from 230 to $276 \mathrm{~cm}$ below surface of mound within

\section{UM-487. Wightman F-8}

$1370 \pm 90$

Charcoal from 322 to $368 \mathrm{~cm}$ below apex of mound within Grid F.

\section{UM-370. Indiantown Sand Mounds}

$$
2110 \pm 50
$$

$160 \mathrm{BC}$

Charcoal from $60 \mathrm{~cm}$ beneath surface of sand mound, Indiantown, Florida $\left(27^{\circ} 02^{\prime} 20^{\prime \prime} \mathrm{N}, 80^{\circ} 34^{\prime} 31^{\prime \prime} \mathrm{W}\right)$. Coll 1974 by M Andrejko; subm 1974 by R Williams, Dept Anthropol, Univ South Carolina. Comment (RW): result used to date bundle burial and early habitation. 


\section{Cannon's Point series}

Shell and wood samples from shell ring, Cannon's Point, St Simon's Island, Glynn Co, Georgia ( $31^{\circ} 16^{\prime} 30^{\prime \prime} \mathrm{N}, 82^{\circ} 20^{\prime} 10^{\prime \prime} \mathrm{W}$ ). Coll 1973-75 and subm 1975 by $\mathrm{R}$ Marrinan.

\section{UM-523. Cannon's Point 2}

$$
3600 \pm 110
$$

Oyster shells from sub-midden humus of Unit Test $\mathrm{I}$ in $\mathrm{W}$ ring (9GN76) 12 to $20 \mathrm{~cm}$ below surface. Comment (RM): dates Iast occupation of site.

\section{UM-521. Cannon's Point 1}

Oyster shells from Unit $18 \mathrm{~N}, 3 \mathrm{E}$ at $20 \mathrm{~cm}$ depth. Comment (RM): dates last occupation of site.

\section{UM-522. Cannon's Point 3}

Oyster shells from lower level of Unit Test I, W ring (9GN76) 45 to $55 \mathrm{~cm}$ below surface. Comment (RM): dates initial occupation of site.

\section{UM-520. Cannon's Point 5}

$4190 \pm 90$

Oyster shell from base of midden deposit $1.47 \mathrm{~m}$ below surface. Comment (RM): dates initial occupation of shell ring and assoc human skeletal material.

\section{UM-519. Cannon's Point 6}

Wood from excavation Sq 27S, 18E, 3.55m below marsh surface, outside of shell ring. Dates transitional period from fiber-tempered ceramics to Deptford ceramics.

\section{UM-518. Cannon's Point 7}

Wood from submarsh floor Excavation Sq 33S, 12E, 3.36 to $3.47 \mathrm{~m}$ below marsh surface. Comment: similar to UM-519.

\section{Arajuno series}

\section{B. Ecuador}

Human bones and skull from grave sites, SE Amazon, Equador ( $\left.1^{\circ} 10^{\prime} 00^{\prime \prime} \mathrm{S}, 78^{\circ} 20^{\prime} 03^{\prime \prime} \mathrm{E}\right)$. Coll 1972 and subm 1975 by P G Turolla.

UM-421. Arajuno Site 1

Modern

Human bones from $3 \mathrm{~m}$ below surface. Comment (PGT): assoc with carved stone artifact. Estimated age: Paleo-Indian. Comment (JJS): inorganic fraction.

UM-422. Arajuno Site 1 AD 1260 
UM-423. Arajuno Site 2

Modern

Human skull from 5m below surface. Comment (PGT): considered Neo-Indian. Comment (JJS): inorganic fraction.

UM-424. Arajuno Site 2

$1650 \pm 70$

Collagen fraction of UM-423. AD 300

\section{Monte Alto series}

\section{Guatemala}

Two charcoal samples from artificial fill under 'Pot Belly' statue, Finca Monte Alto, La Democracia, Guatemala $\left(14^{\circ} 13^{\prime} 20^{\prime \prime} \mathrm{N}, 90^{\circ} 56^{\prime}\right.$ 30" W). Coll 1970 and subm 1974 by E M Shook, Antigua, Guatemala. General Comment (EMS): samples are possibly from cooking or pottery making fires. Age of this culture is difficult to determine since assoc of monuments and pottery are not indigenous to area of find. Results indicate emplacement of statuary during Pre-Classic era. Emplacement corresponds chronologically with 'Pot Belly' emplacement at Finca Santa Leticia, El Salvador (R, v 18, p 116).

\section{UM-389. Monte Alto M44 W to Z}

$$
2450 \pm 90
$$

$500 \mathrm{BC}$

\section{UM-621. Monte Alto M8 K3}

$$
\begin{aligned}
& 2020 \pm 70 \\
& 70 \mathrm{BC}
\end{aligned}
$$

D. Honduras

\section{Port Royal series}

Two samples from each of 2 shipwrecks (PRI \& PR4) lying underwater at $\mathrm{E}$ end of Port Royal Bay, Isla de Roatan, Islas de la Bahia, Honduras $\left(16^{\circ} 24^{\prime} 20^{\prime \prime} \mathrm{N}, 86^{\circ} 15^{\prime} 26^{\prime \prime} \mathrm{W}\right)$. Coll and subm 1975 by $\mathrm{J} \mathrm{E}$ Hall, Dept Anthropol, Univ Miami.

General Comment (JEH): wrecks were covered with sand and turtle grass in water depth ca $9 \mathrm{~m}$. Ships were thought to pre-date Columbus, AD 1492.

UM-625. Port Royal PR1-6

Charcoal from front hold sec.

UM-626. Port Royal PR1-7

Carbonized wood from front hold sec. Comment (JJS): average age for UM-625 \& UM-626 is $245 \pm 45$. Samples from same wreck were previously dated by Pennsylvania ( $R, v 16, \mathrm{p} 23)$.

\section{UM-622. Port Royal PR4-1}

Wood sample from Sec 13G thought to be centerboard.
$230 \pm 70$

AD 1720

$260 \pm 60$

$130 \pm 60$ 
UM-623. Port Royal PR4-1

Comment (JJS): duplicate run of UM-622. Average age is $140 \pm 40$.

UM-624. Port Royal PR4-2

Modern

Wood sample taken from Sec 15G thought to be centerboard.

II. GEOLOGIC SAMPLES

A. United States

Caesars Creek Bank series

Shell samples from 2 piston cores in .7 to $1 \mathrm{~m}$ water, Caesars Creek Bank, Biscayne Bay, Florida. Combined carbonate mudbank and tidal flats assoc with major tidal pass between Biscayne Bay and inner-reef tract SE coast of Florida. Dates depositional sequence of bank. Coll and subm 1975 by E R Warzeski, RSMAS, Univ Miami. Core 674 from $\left(25^{\circ} 23^{\prime} 53^{\prime \prime}\right.$ N, $\left.80^{\circ} 13^{\prime} 01^{\prime \prime} \mathrm{W}\right)$. Core 575 from $\left(25^{\circ} 22^{\prime} 53^{\prime \prime} \mathrm{N}, 80^{\circ} 12^{\prime} 56^{\prime \prime} \mathrm{W}\right)$. See also (R, v 18, p 117-119).

\section{UM-552. Core W-674-1D}

$4210 \pm 140$

Assorted shells (primarily Modiolus americanus), 428 to $448 \mathrm{~cm}$ below sediment surface.

\section{UM-553. Core W-674-1E}

$5480 \pm 140$

3530 BC

Assorted shells (primarily Anodontia alba, Astrea tecta americana), 400 to $440 \mathrm{~cm}$ below sediment surface.

\section{UM-525. Core W-575-1A}

$4220 \pm 110$

Large mollusk shells (Laevicardium laevigatum, Astrea phoebia, and Tellina lineata), 390 to $420 \mathrm{~cm}$ below sediment surface.

\section{UM-526. Core W-575-1B}

$4230 \pm 140$

Small mollusk shells, bivalves and gastropods, 380 to $420 \mathrm{~cm}$ below sediment surface.

\section{UM-551. Core W-575-1C}

$5120 \pm 140$

$3170 \mathrm{BC}$

Assorted shells (primarily Modiolus americanus and Astrea phoebia), 440 to $455 \mathrm{~cm}$ below sediment surface.

\section{Shackleford Banks series}

Samples from split-spoon cores on Shackleford Banks, Carteret Co, North Carolina, between ( $\left.34^{\circ} 39^{\prime} 28^{\prime \prime} \mathrm{N}, 76^{\circ} 33^{\prime} 50^{\prime \prime} \mathrm{W}\right)$ and $\left(34^{\circ} 41^{\prime} 07^{\prime \prime}\right.$ N, $\left.76^{\circ} 38^{\prime} 45^{\prime \prime} \mathrm{W}\right)$. Coll 1973 and subm 1975 by K Susman, Duke Univ. See also (R, v 17, p 239).

General Comment (KS): dates stratigraphic sequence and local buried geomorphic features for Shackleford Banks. 
UM-576. Shackleford Sh-3,18

$>\mathbf{3 6 , 3 2 0}$

Mercenaria shells from $20.8 \mathrm{~m}$ depth. Comment (KS): may be part of a Tertiary lag erosion surface.

\section{UM-581. Shackleford Sh-4,14}

$14,880 \pm 570$

12,930 вC

Nuculana, Arca, and Mulinia shells from 15.2 to $15.8 \mathrm{~m}$ depth. Comment (KS): mud sediment lies below sand recognized as inlet-filling sand. Question was whether this is Holocene transgression or Wisconsin mud.

\section{UM-577. Shackleford Sh-5,20}

$$
23,590 \pm 620
$$

Shell of unid. species in fine sand at $23.2 \mathrm{~m}$ depth. Comment (KS): underlies a semi-indurated (Tertiary?) limestone.

\section{UM-579. Shackleford Sh-8,13}

$$
26,950
$$

25,000 BC

Mulinia, Crassostrea virginica, and Arca shells. Comment (KS): in sandy, silty clay believed to be a Pleistocene backbarrier sediment.

UM-582. Shackleford Sh-8,18

Mercenaria shell from $22.7 \mathrm{~m}$ depth. Comment (KS): helps date clay units that bracket shell-and-pebble lag.

UM-574. Shackleford Sh-11,7

$$
470 \pm 60
$$

\section{AD 1480}

Peat. Comment (KS): located in what should be outcrop of marsh peat on front of barrier island.

$$
+2000
$$

UM-580. Shackleford Sh-11,16

$27,330 \mathrm{BC}$

Mulinia, Tegolus, and Arca shells. Comment (KS): thought to be Pleistocene backbarrier bay mud.

\section{UM-578. Shackleford Sh-13,14}

$11,270 \pm 170$

9320 BC

Unid. shell from $14 \mathrm{~m}$ depth in matrix of sand. Comment (KS): part of a beach or inlet sequence.

\section{UM-575. Shackleford Sh-13,19}

Unid. shell species from depth $21.6 \mathrm{~m}$. Matrix of sand. Comment (KS): thought to be former channel fill.

\section{New Jersey Shelf series}

Samples cored on secs of New Jersey shelf. Coll June 1974 by G L Freeland; subm Dec 1974 by WL Stubblefield, NOAA, Miami, Florida. 
UM-416. New Jersey Shelf, 1A-V6-50

Shell material near shore from depth $50 \mathrm{~cm}\left(39^{\circ} 25^{\prime} \mathrm{N}, 74^{\circ} 20^{\prime} \mathrm{W}\right)$. Comment (WLS): to date late aggradation of flank of inner ridge crest.

UM-417. New Jersey Shelf, 1A-V6-563-573

AD 1280

$670 \pm 70$

Shell material from 563 to $573 \mathrm{~cm}$ depth in actively reworked Holocene sand on inner ridge crest $\left(39^{\circ} 25^{\prime} \mathrm{N}, 74^{\circ} 20^{\prime} \mathrm{W}\right)$. Comment (WLS): dates ridge aggradation.

\section{UM-412. New Jersey Shelf, 1B-V5-35}

$3980 \pm 600$

Shell material from $35 \mathrm{~cm}$ depth in hydraulically active Holocene sediment $\left(39^{\circ} 08^{\prime} \mathrm{N}, 74^{\circ} 35^{\prime} \mathrm{W}\right)$. Comment (WLS): late ridge aggradation in central ridge/swale system.

\section{UM-418. New Jersey Shelf, 1B-V7-60}

$5600 \pm 130$ $3650 \mathrm{BC}$

Shell hash from $60 \mathrm{~cm}$ depth in hydraulically active Holocene sediment $\left(39^{\circ} 08^{\prime} \mathrm{N}, 74^{\circ} 05^{\prime} \mathrm{W}\right)$. Comment (WLS): represents later flank aggradation on central shelf ridge.

\section{UM-415. New Jersey Shelf, 1B-V7-221}

$4370 \pm 250$ 2420 BC

Shell material from $221 \mathrm{~cm}$ depth in recent Holocene sediment $\left(39^{\circ}\right.$ $08^{\prime} \mathrm{N}, 74^{\circ} 05^{\prime} \mathrm{W}$ ). Comment (WLS): results determine amount of flank aggradation.

\section{UM-414. New Jersey Shelf, 1B-V9-20}

$$
800 \pm 60
$$

Shell material from $20 \mathrm{~cm}$ depth in central shelf trough $\left(39^{\circ} 08^{\prime} \mathrm{N}\right.$, $74^{\circ} 05^{\prime} \mathrm{W}$ ). Comment (WLS): expected to date development of sand ridges on inner central shelf.

\section{UM-413. New Jersey Shelf, 1B-V10-2}

$2070 \pm 130$

Shell material from $2 \mathrm{~cm}$ depth in hydraulically active substrate from Holocene lagoon $\left(39^{\circ} 08^{\prime} \mathrm{N}, 74^{\circ} 05^{\prime} \mathrm{W}\right)$. Comment (WLS): dates base of a ridge system.

\section{UM-419. New Jersey Shelf, 1B-V10-40}

$3980 \pm 190$ 2030 BC

Shell material from $40 \mathrm{~cm}$ depth in Pleistocene sand of central trough area $\left(39^{\circ} 08^{\prime} \mathrm{N}, 74^{\circ} 05^{\prime} \mathrm{W}\right)$. Comment (WLS): lower constraining date of Holocene lagoonal sediment.

\section{Angelfish Creek series}

Peat sequence from vertical exposure in wall of tidal pass, $\mathrm{N}$ Florida Keys, Florida $\left(25^{\circ} 20^{\prime} \mathrm{N}, 80^{\circ} 17^{\prime} \mathrm{W}\right)$. Coll 1975 by R B Halley; subm 1975 by E A Shinn, USGS, Fisher I. sta, Miami, Florida. 
UM-584. Angelfish Creek, $100 \mathrm{~cm}$

$2090 \pm 90$

UM-584. Angelfish Creek, $100 \mathrm{~cm}$

UM-585. Angelfish Creek, $150 \mathrm{~cm}$

140 BC

$2650 \pm 90$

$700 \mathrm{BC}$

UM-586. Angelfish Creek, $200 \mathrm{~cm}$

$2850 \pm 60$ $900 \mathrm{BC}$

UM-587. Angelfish Creek, $250 \mathrm{~cm}$

$3170 \pm 70$

1220 BC

UM-588. Angelfish Creek, $300 \mathrm{~cm}$

$3710 \pm 70$

$1760 \mathrm{BC}$

UM-589. Angelfish Creek, $350 \mathrm{~cm}$

$3970 \pm 100$

2020 BC

UM-590. Angelfish Creek, $400 \mathrm{~cm}$

$4670 \pm 70$

2720 BC

UM-591. Angelfish Creek, $450 \mathrm{~cm}$

$4150 \pm 150$

2200 BC

UM-592. Angelfish Creek, $480 \mathrm{~cm}$

$4220 \pm 80$

2270 BC

UM-593. Angelfish Creek, $490 \mathrm{~cm}$

$4800 \pm 100$

2850 BC

Sands Cut series

Peat sequence from vertical exposure in channel wall at Sands Cut, N Florida Keys, Florida $\left(25^{\circ} 28^{\prime} \mathrm{N}, 80^{\circ} 10^{\prime} \mathrm{W}\right)$. Coll 1975 by R B Halley; subm 1975 by E A Shinn. Fisher I. sta.

General Comment (EAS): dates sea level change.

UM-607. Sands Cut, $0 \mathrm{~cm}$ AD 1590

$360 \pm 60$

UM-608. Sands Cut, $10 \mathrm{~cm}$

$4160 \pm 140$ 2210 BC

UM-609. Sands Cut, $50 \mathrm{~cm}$ $4080 \pm 90$ $2130 \mathrm{BC}$

UM-610. Sands Cut, $100 \mathrm{~cm}$ $3980 \pm 80$ 2030 BC

UM-611. Sands Cut, $150 \mathrm{~cm}$ $2580 \pm 70$ 630 BC

UM-612. Sands Cut, $200 \mathrm{~cm}$ $2530 \pm 80$ 580 BC 
UM-613. Sands Cut, $200 \mathrm{~cm}$

\section{UM-594. Fort Lauderdale Reef}

Coral (A palmata) sawed from dead sec of reef in $4.5 \mathrm{~m}$ water off coast of Fort Lauderdale, Florida $\left(26^{\circ} 08^{\prime} 15^{\prime \prime} \mathrm{N}, 80^{\circ} 05^{\prime} 05^{\prime \prime} \mathrm{W}\right)$. Coll 1975 by W Raymond, Britt Assocs, Miami; subm 1975 by E A Shinn. Comment (EAS): result to determine when this major reef builder died in area $\mathrm{N}$ of Miami. Estimated age: $<200 \mathrm{yr}$.

\section{Mt Pelée series}

B. Martinique, West Indies

Charcoal from pyroclastic surge sediments near Mt Pelée, Martinique, West Indies. Dates used to determine frequency of cyclic eruptions on Mt Pelée. Coll and subm by J Roobol and A L Smith, Univ Puerto Rico at Mayaguez.

\section{UM-376. Pelée 211}

$2670 \pm 70$

Sample in block ash sediment in $720 \mathbf{~ B C}$ of Mt Pelée $\left(14^{\circ} 48^{\prime} 26^{\prime \prime} \mathrm{N}, 61^{\circ} 05^{\prime} 51^{\prime \prime} \mathrm{W}\right)$.

\section{UM-377. Pelée 332}

$5190 \pm 90$

Sample from dense laval in rd sec near Rivière Calava, SW Mt Pelée (14 $46^{\prime} 22^{\prime \prime} \mathrm{N}, 61^{\circ} 08^{\prime} 47^{\prime \prime} \mathrm{W}$ ).

\section{UM-378. Pelée 332}

$5310 \pm 120$

Duplicate run of UM-377. Comment (JJS): average age of UM-377 and -378 is $5250 \pm 70$.

\section{UM-379. Pelée 331}

$8400 \pm 210$

$\mathbf{6 4 5 0} \mathbf{~ B C}$
Sample from block and ash sediments in rd cut near Rivière Balisier $\left(14^{\circ} 46^{\prime} 33^{\prime \prime} \mathrm{N}, 61^{\circ} 08^{\prime} 45^{\prime \prime} \mathrm{W}\right)$. Comment (ALS): important in correlating complex sequence of surge and pumice flow sediments from $S$ Pelée.

\section{UM-380. Pelée 381}

$$
440 \pm 120
$$

\section{AD 1510}

Sample from block and ash sediment of limited areal extent. Forms low banks in river bed, middle sec Rivière des Pères $\left(14^{\circ} 46^{\prime} 54^{\prime \prime} \mathrm{N}, 61^{\circ}\right.$ $\left.10^{\prime} 00^{\prime \prime} \mathrm{W}\right)$. Comment (ALS): possibly represents latest prehistoric erup-
tion.

\section{UM-382. Pelée 381}

$$
540 \pm 110
$$

Duplicate run of UM-380. Comment (JJS): average age of UM-380 and -382 is $490 \pm 80 \mathrm{yr}$. 
UM-381. Pelée 448

$1040 \pm 90$

Sample from dense lava surge deposit, cliff sec, lower part of gorge of Rivière Claire (14 $\left.46^{\prime} 09^{\prime \prime} \mathrm{N}, 61^{\circ} 11^{\prime} 42^{\prime \prime} \mathrm{W}\right)$.

UM-410. Pelée 455

$4020 \pm 80$

Sample from block and ash sediments, cliff sec, lower part of gorge of Rivière Claire (14 $\left.46^{\prime} 46^{\prime \prime} \mathrm{N}, 61^{\circ} 11^{\prime} 09^{\prime \prime} \mathrm{W}\right)$.

\section{UM-411. Pelée 455}

$3990 \pm 80$

Duplicate run of UM-410. Comment (JJS): average age of UM-410 and -411 is $4005 \pm 57 \mathrm{yr}$.

\section{UM-383. Pelée 302}

$25,120 \pm 450$

\section{3,170 вC}

Sample from block and ash sediment, cliff sec in lower part of Rivière Precheur (14 $\left.48^{\prime} 18^{\prime \prime} \mathrm{N}, 61^{\circ} 13^{\prime} 26^{\prime \prime} \mathrm{W}\right)$. Comment (ALS): stratigraphically one of oldest deposits containing carbon.

\section{UM-384. Pelée 450}

$2470 \pm 80$

520 BC

Sample from pumice flow cloud deposit, in rd to Morne Cocos $\left(14^{\circ}\right.$ $49^{\prime} 09^{\prime \prime} \mathrm{N}, 61^{\circ} 13^{\prime} 39^{\prime \prime} \mathrm{W}$ ). Comment (ALS): important for stratigraphy of NW sector of Mt Pelée.

UM-385. Pelée 450

$2430 \pm 80$

$480 \mathrm{BC}$

Duplicate run of UM-384. Comment (JJS): average age of UM-384 and -385 is $2450 \pm 57 \mathrm{yr}$.

\section{UM-386. Pelée 388A}

34,150 BC

Sample from dense lava surge deposit in rd cut between Anse Belloville and Anse Ceron (14 49 $13^{\prime \prime}$ N, $\left.61^{\circ} 13^{\prime} 53^{\prime \prime} \mathrm{W}\right)$.

\section{UM-387. Pelée 344}

$7410 \pm 130$

Sample from pumice flow sediment in cliff sec near Macouba, Martinique $\left(14^{\circ} 50^{\prime} 14^{\prime \prime} \mathrm{N}, 61^{\circ} 09^{\prime} 08^{\prime \prime} \mathrm{W}\right)$. Comment (ALS): only carbon sample from $\mathrm{E}$ side of volcano.

UM-426. Pelée 222

$2150 \pm 70$

$200 \mathrm{BC}$

Sample from pumice flow sediment in cliff sec, lower part of Rivière Pointe la Mare (14 46 $\left.57^{\prime \prime} \mathrm{N}, 61^{\circ} 12^{\prime} 50^{\prime \prime} \mathrm{W}\right)$. Comment (ALS): one of most important pumice flow sediments on W Pelée. 
UM-427. Pelée 245

$4230 \pm 120$

3280 BC

Sample from block and ash sediment on rd between Pointe La Mare

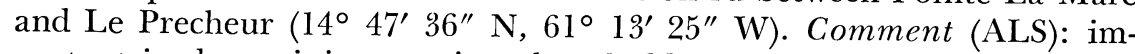
portant in determining stratigraphy of older deposits on $\mathrm{W}$.

\section{UM-428. Pelée 310}

$20,240 \pm 610$ $18,290 \mathrm{BC}$

Sample from block and ash sediment in cliff alongside rd between Le Precheur and Anse Belleville (14 48 $23^{\prime \prime}$ N, $61^{\circ} 13^{\prime} 52^{\prime \prime}$ W).

UM-429. Pelée 311

Sample from pumice flow in cliff and gully sec along rd immediately $\mathrm{N}$ of Le Precheur $\left(14^{\circ} 48^{\prime} 20^{\prime \prime} \mathrm{N}, 61^{\circ} 13^{\prime} 50^{\prime \prime} \mathrm{W}\right)$.

\section{UM-430. Pelée 410}

$310 \pm 60$

Sample from block and ash sediment in $S$ bank near mouth of Rivière des Pères ( $\left.14^{\circ} 45^{\prime} 12^{\prime \prime} \mathrm{N}, 61^{\circ} 11^{\prime} 03^{\prime \prime} \mathrm{W}\right)$.

UM-431. Pelée 439

$2560 \pm 70$

$610 \mathrm{BC}$

Sample from pumice flow cloud deposit in quarry on $S$ bank near mouth of Rivière Seche $\left(14^{\circ} 45^{\prime} 43^{\prime \prime} \mathrm{N}, 61^{\circ} 11^{\prime} 43^{\prime \prime} \mathrm{W}\right)$. Comment (ALS): important for correlation of late-prehistoric pumice flow sediments. UM432 in sediment below but separated by marked unconformity.

UM-432. Pelée 440

$3940 \pm 80$

1990 BC

Sample from dense lava surge sediment in quarry on $S$ bank near mouth of Rivière Seche $\left(14^{\circ} 45^{\prime} 43^{\prime \prime} \mathrm{N}, 61^{\circ} 11^{\prime} 43^{\prime \prime} \mathrm{W}\right)$. See also UM-431.

\section{UM-433. Pelée 446}

$1140 \pm 70$

Sample from thick airfall sediment in cliff sec, upper part of Rivière Seche $\left(14^{\circ} 47^{\prime} 11^{\prime \prime} \mathrm{N}, 61^{\circ} 10^{\prime} 46^{\prime \prime} \mathrm{W}\right)$. Comment (ALS): only carbon obtained from an airfall sediment.

\section{REFERENCES}

Eldridge, K L, Stipp, J J, and Cohen, S J, 1975, University of Miami radiocarbon dates III: Radiocarbon, v 17, p 239-246.

Eldridge, K L, Stipp, J J, and Hattner, J, 1975, University of Miami radiocarbon dates V: Radiocarbon, v 18, p 116-124. Lawn, Barbara, 1974, University of Pennsylvania radiocarbon dates XVII: Radiocarbon,
v 16, p 231.

Stipp, J J, Eldridge, K L, Cohen, S J, and Webber, K, 1974, University of Miami radiocarbon dates I: Radiocarbon, v 16 , p 402-408.

Tamers, M A, 1975, Chemical yield optimization of the benzene synthesis for radiocarbon dating: Internatl Jour Appl Rad \& Isotopes, v 26, p 676-682. 